\title{
Prótesis de cuerpo vertebral JR: dispositivo modular, anatómico y expandible, con función de jaula y placa diseñada ad hoc para estabilizar la columna después de corpectomía
}

Prótese do corpo vertebral JR: dispositivo modular, anatômico e expansível, com função de jaula e placa desenhada ad hoc para estabilizar a coluna depois da corpectomia

JR vertebral body prosthesis: a modular, anatomical and expandable device, with cage function and plate dual designed ad hoc for spine stabilization after corpectomy

\author{
Juan José Ramírez Jiménez \\ Erwin Chiquete ${ }^{2}$ \\ Silvia Ramírez ${ }^{3}$ \\ Rafael Guerrero ${ }^{4}$
}

\section{RESUMEN}

Introducción: debido a los resultados clínicos no satisfactorios con instrumentaciones posteriores en los casos de tumor vertebral y a las carencias económicas de nuestros pacientes para adquirir los sistemas disponibles en el mercado, fue diseñada una prótesis de cuerpo vertebral para estabilizar la columna después de una corpectomía. Objetivo: describir las características estructurales y funcionales de la prótesis JR, las pruebas biomecánicas en cadáver y la técnica quirúrgica en el paciente. Métodos: primeramente fue realizado un estudio anatómico detallado de los cuerpos vertebrales. Se obtuvo el diseño de un dispositivo modular, anatómico y expandible. Sus componentes una vez ensamblados hacen un implante con

\section{RESUMO}

Introdução: devido aos resultados clínicos não satisfatórios com instrumentações posteriores nos casos de tumor vertebral e às carências econômicas dos nossos pacientes para adquirir os sistemas disponíveis no mercado, foi desenhada uma prótese de corpo vertebral para estabilizar a coluna depois de uma corpectomia. Objetivo: descrever as características estruturais e funcionais da prótese JR, as provas biomecânicas no cadáver e a técnica cirúrgica no paciente. Métodos: primeiro foi realizado um estudo anatômico detalhado dos corpos vertebrais. Obteve-se o desenho de um dispositivo modular, anatômico e expansível. Seus componentes uma vez ensamblados fazem um implante com função dual de jaula

\begin{abstract}
Introduction: due to the unsatisfactory results obtained with posterior instrumentations in cases of vertebral tumors and to the economical limitations of our patients for acquiring the systems available in the market, a vertebral body prosthesis to stabilize the spine after corpectomy was designed. Objective: to describe the structural and functional characteristics of the JR prosthesis, the biomechanical assays in cadaver and the surgical technique on the patient. Methods: firstly, it was performed a detailed anatomical study of the vertebral bodies. A design of a modular, anatomical and expandable device was obtained. Its components once assembled make an implant with expandable cage and plate dual
\end{abstract}

Servicio de Ortopedia del Hospital Civil de Guadalajara "Fray Antonio Alcalde" de la Universidad de Guadalajara - Guadalajara, México.

"MD del Servicio de Ortopedia del Hospital Civil de Guadalajara "Fray Antonio Alcalde" de la Universidad de Guadalajara - Guadalajara, México.

2PhD; MD del Servicio de Medicina Interna del Hospital Civil de Guadalajara "Fray Antonio Alcalde" de la Universidad de Guadalajara - Guadalajara, México. ${ }^{3} \mathrm{MD}$ del Departamento de Enseñanza e Investigación del Hospital Civil de Guadalajara "Fray Antonio Alcalde" de la Universidad de Guadalajara - Guadalajara, México. ${ }^{4} \mathrm{MD}$ del Servicio de Neurología y Neurocirugía del Hospital Civil de Guadalajara "Fray Antonio Alcalde" de la Universidad de Guadalajara - Guadalajara, México. No hay conflicto de intereses. 
función dual de jaula y placa lateral expandibles. Posteriormente se efectuó un ensayo de propiedades biomecánicas en cadáver y se implantó un dispositivo en un paciente con metástasis en cuerpo vertebral. Resultados: las radiografías que le fueron tomadas al cadáver, posterior a la colocación del implante, no mostraron pérdida de la fijación. Al levantar el cadáver se generaron momentos de flexión en sentido lateral con brazos de palanca de $80 \mathrm{~cm}$ de largo, por lo que la prótesis fue demandada en su punto más vulnerable con una fuerza de aproximadamente $588 \mathrm{~N}$. Con la rotación, flexión y extensión forzadas, la estabilidad se conservó y no se visualizó movimiento del implante. Se colocó el dispositivo en una mujer de 50 años con cáncer metastásico de tiroides que afectaba L3. El dolor mejoró en el postoperatorio inmediato, así como su función motora que le permitió caminar con una columna estable e indolora por siete años. No se observó fracaso del implante. Conclusión: la función dual de jaula y placa integrada en la prótesis de cuerpo vertebral establece una ventaja mecánica comparada con la función de la jaula y de la placa separadamente, ya que el diseño de la prótesis permite aplicar fuerza axial y fijación lateral al mismo tiempo, a través de solo un implante. Los resultados de la colocación del implante en paciente son satisfactorios.

DESCRIPTORES: Prótesis e implantes; Placas óseas; Columna vertebral/ cirugía; Procedimientos ortopédicos/instrumentación; Procedimientos ortopédicos/ métodos e placa lateral expansível. Posteriormente, realizou-se um ensaio de propriedades biomecânicas em cadáver e se implantou um dispositivo num paciente com metástase no corpo vertebral. Resultados: as radiografias que foram tiradas do cadáver, posterior à colocação do implante não mostraram perda da fixação. Ao levantar o cadáver se geraram momentos de flexão no sentido lateral com braços de palanca de $80 \mathrm{~cm}$ de longitude, neste caso a prótese foi desafiada no seu ponto mais vulnerável com uma força de aproximadamente 588 N. Com a rotação, flexão e extensão forçadas, a estabilidade se conservou e não foram visualizados movimentos do implante. Colocou-se o dispositivo numa mulher de 50 anos com câncer metastático de tiroides que afeitava L3. A dor melhorou nos pósoperatório imediato, assim como sua função motora, a qual permitiu que a paciente caminhasse com uma coluna estável e indolor por sete anos. Não se observou fracasso do implante. Conclusão: a função dual de jaula e placa integrada na prótese de corpo vertebral estabelece uma vantagem mecânica comparada com a função de jaula e placa separadamente, uma vez que o desenho da prótese permite aplicar força axial e fixação lateral ao mesmo tempo, por meio de um só implante. Os resultados da colocação do implante no paciente foram satisfatórios. function. Afterwards, a biomechanical assay was performed in a cadaver and the device was implanted in a patient with vertebral body metastasis. Results: the radiographs extracted from the cadaver after implant location did not show loss of fixation. When the cadaver was raised, flexion moments were generated laterally with levers 80 $\mathrm{cm}$ long; therefore, the prosthesis was demanded in its most vulnerable point with a force of approximately $588 \mathrm{~N}$. With rotation, flexion and extensions forced, stability was preserved and no movement was observed. The device was implanted in a 50-year-old woman with metastatic thyroid cancer affecting L3. Pain improved in the postoperative immediate, as well as its motor function, which allowed her to walk with a stable and painless spine for seven years. No implant failure was observed. Conclusion: the dual cage function and plate integrated in a single device offers a mechanical advantage, compared with the cages and plates used separately, since the prosthesis design permits to apply a distraction axial force with lateral fixation at the same time, through one implant. The results of the implant placement in the patient were satisfactory.

KEYWORDS: Prostheses and implants; Bone plates; Spine/surgery; Orthopedic procedures/instrumentation; Orthopedic procedures/ methods

\section{INTRODUCCIÓN}

Paul Harrington en la década de 1950 y Eduardo Luque en la de 1970, diseñaron sus implantes homónimos para evitar las complicaciones de los yesos en sus pacientes que trataban de escoliosis. Subsecuentemente estos implantes se extendieron a fracturas, tumores y problemas degenerativos $^{1,2}$. Hasta la década de 1990, en nuestro hospital los pacientes con fractura o tumor de cuerpo vertebral que no respondían a otras formas de terapia eran tratados con laminectomía e instrumentación posterior con estos sistemas, sin embargo la mayoría de los pacientes no aliviaba su dolor y el déficit neurológico persistía. Frustrados con los resultados clínicos obtenidos, se diseñó un implante para usar como prótesis de cuerpo vertebral, con el objetivo de aliviar el dolor, estabilizar la columna y preservar o restaurar la función neurológica. Previo a la colocación 
en pacientes, este implante fue sometido a una prueba de fijación en cadáver.

El objetivo de este artículo es describir la prótesis vertebral JR en relación a su estructura y función, así como reportar los resultados de las pruebas de propiedades biomecánicas, según un ensayo en cadáver. Se reporta además un caso clínico ejemplar, en el que se ha usado el implante para estabilizar la columna después de corpectomía.

\section{MÉTODOS}

\section{Diseño del implante}

En el Departamento de Anatomía de la Universidad de Guadalajara, fueron medidos los cuerpos vertebrales de las vertebras disponibles, exceptuando $\mathrm{C} 1$ y $\mathrm{C} 2$, en relación a su altura anterior y posterior, diámetro sagital y coronal. Se buscó y se determinó el centro de la curva anterior y laterales izquierda y derecha de los cuerpos vertebrales de la región cervical, torácica, toracolumbar y lumbar. Además, en radiografías simples de la columna vertebral en proyección AP se midió la inclinación que va teniendo la columna en dirección de caudal a cefálica, partiendo desde L5 y midiéndolo cada tres segmentos hasta llegar a la región torácica alta. Con los datos anatómicos obtenidos, se hicieron dibujos y se estimaron las dimensiones de las prótesis, posteriormente se hicieron modelos en yeso y cera. Finalmente, con la técnica de la "cera perdida", se hizo el primer prototipo en cromo-cobalto, luego un segundo en acero inoxidable y un tercero en titanio (Figura 1).

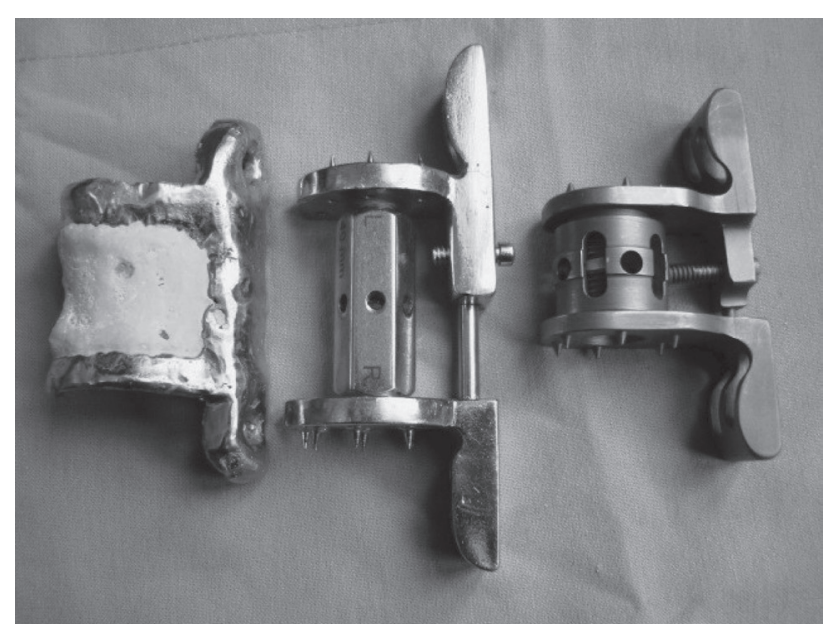

Figura 1

Evolución de los prototipos de la prótesis de cuerpo vertebral JR: cromio-cobalto (izquierdo), acero inoxidable (medio) y titanio (derecho).

\section{Descripción de la prótesis JR}

La prótesis vertebral JR, diseñada en el Servicio de Ortopedia del Hospital Civil de Guadalajara "Fray Antonio Alcalde" es anatómica, modular y expandible. Está formada por cinco componentes: componente cefálico, componente caudal, cilindro central, perno guía anti-rotacional y tornillo opresor (Figura 2). Sus componentes una vez en- samblados forman una placa lateral y una jaula cilíndrica (Figura 3). El elemento horizontal de ambos componentes presenta proyecciones cónicas en su superficie de sustentación para aumentar la fijación y evitar el cizallamiento entre el implante y la vértebra. Además, es presentado un orificio en su centro para que permita puentear biológicamente la vértebra de arriba con la de abajo mediante un relleno de injerto óseo. Este orificio se continúa en ambos componentes a través del elemento central, lo cual esta roscado en su exterior con rosca derecha e izquierda, respectivamente, de tal forma que rotando el cilindro central (componente C), en dirección opuesta a las manecillas del reloj los componentes cefálico y caudal se alejen, y viceversa se aproximen. De esta forma se hace la jaula cilíndrica expandible. La placa lateral está dispuesta en el lado derecho de la prótesis para la región torácica alta y media; y en el lado izquierdo para la región torácica baja, toracolumbar y lumbar hasta L3. La parte que hace placa lateral en el componente cefálico presenta en su parte inferior un orificio para dar alojamiento al perno guía antirotacional (componente D), este se une en el componente caudal mediante rosca para evitar la rotación entre ambos componentes (Figura 4). Estos elementos verticales presentan en sus extremos dos orificios para fijación lateral a la vértebra correspondiente. De esta forma, se hace la placa lateral expandible y se evita la rotación entre el implante y la columna.

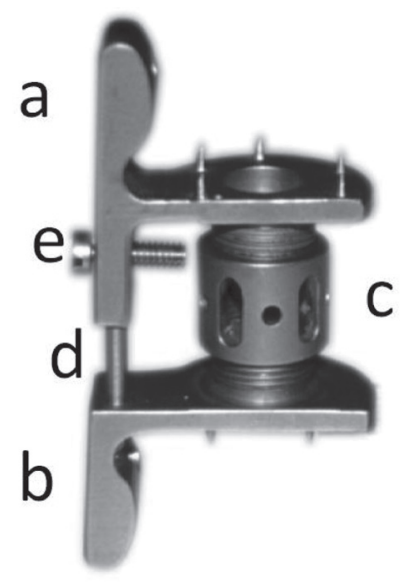

Figura 2

Componentes de la prótesis de cuerpo vertebral JR: a) componente cefálico, b) componente caudal, c) cilindro central, d) perno guía anti-rotacional y e) tornillo opresor.

\section{Biomecánica de la prótesis JR}

Por su diseño modular, anatómico y expandible, su localización anterior al centro de rotación y su función de jaula y placa (distracción axial y fijación lateral) ofrecen vectores opuestos y cruzados a los momentos de flexión, extensión y rotación de la columna. Además, debido a que la forma geométrica y el radio de los elementos horizontales (área de sustentación) de ambos componentes son aproximadamente iguales al área y el radio de las placas terminales de las vértebras que sustenta, recrea el momento inercial, lo 


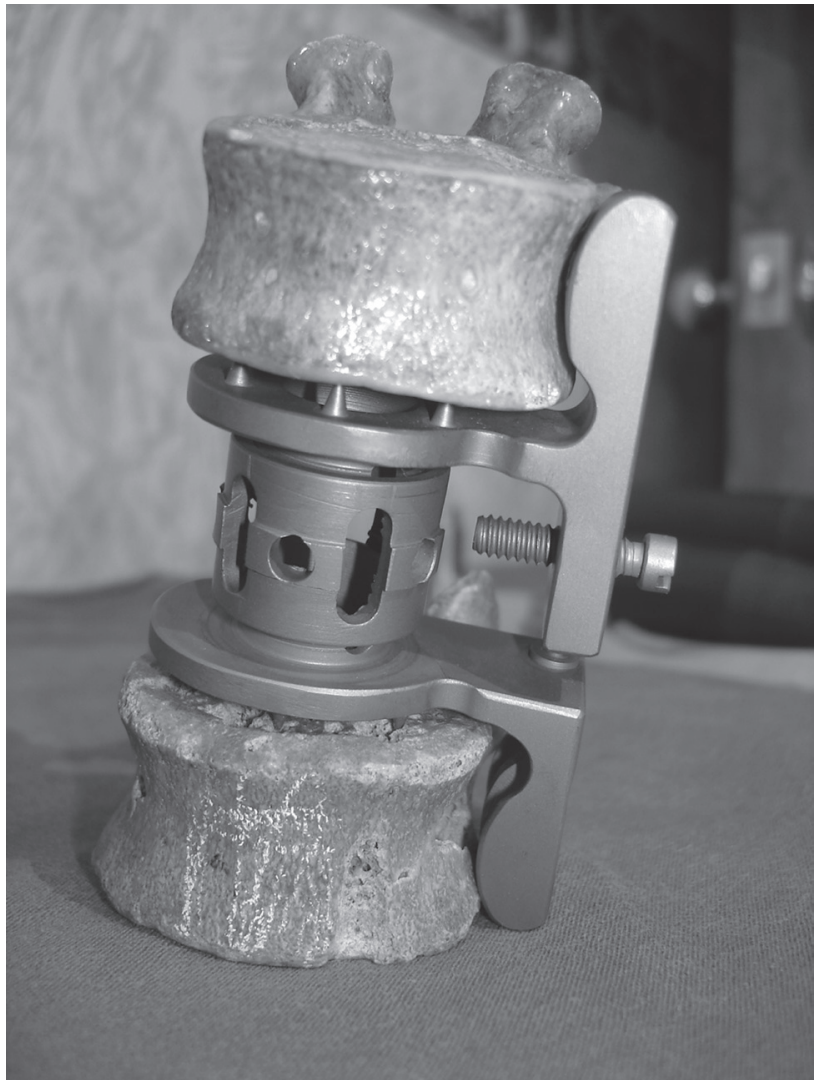

Figura 3

Prótesis de cuerpo vertebral. JR: jaula y placa expandibles integrada en un solo implante.

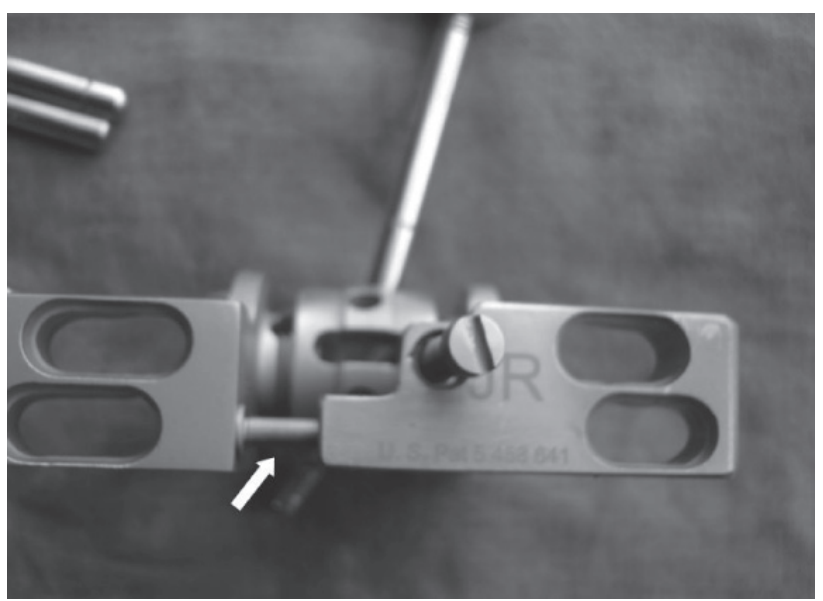

Figura 4

Perno guía anti-rotacional que se inserta en el orificio inferior del elemento vertical del componente cefálico (flecha), para evitar rotación entre sus componentes.

cual aumenta no sólo su resistencia a la inclinación sino que también aumenta la ventaja mecánica del diseño para la estabilización de la columna, aumentando la vida del implante, ya que disminuye la falla por fatiga. El momento de inercia es una propiedad geométrica relacionada al área de sección transversal de una estructura. Esta describe la distribución espacial del material en una sección de la estructura con relación a su eje neutro y no tiene relación con el material con el que está hecho; en pocas palabras, establece la habilidad del material para resistir inclinación.

Esta resistencia a la inclinación se incrementa a la cuarta potencia del radio de la estructura. Es decir, un pequeño cambio en el diámetro de una varilla tiene un gran efecto en la resistencia a la inclinación. El momento de inercia es proporcional a la cuarta potencia del radio de la estructura ${ }^{3}$.

$$
\mathrm{I}=1 / 4(\pi) \mathrm{r}^{4}
$$

Más aún, en el equilibrio estático, las fuerzas y los momentos causan cambio en la forma de la estructura. Sin embargo, por la base amplia de ambos componentes de la prótesis JR es que se disminuye el estrés sobre las placas terminales, ya que estrés es igual a fuerza sobre área $(\sigma=F / A)$. Por eso, las posibilidades de hundimiento del implante disminuyen. A mayor área menor estrés y a menor estrés menor deformidad (strain).

\section{Ensayo de propiedades mecánicas en cadáver}

Los materiales usados fueron: prótesis de cuerpo vertebral $\mathrm{JR}$, cuatro tornillos $6.5 \mathrm{~mm}$, esponjosa rosca corrida de 45 $\mathrm{mm}$, dinamómetro, dos poleas, cuerdas, aparato de rayos $\mathrm{X}$, cadáver como espécimen biológico, instrumental de cirugía general, instrumental para corpectomía (martillo, osteotomo, pinzas gubias, pinzas de disco), instrumental para la colocación de la prótesis (martillo, punzón, desarmador hexagonal $4.5 \mathrm{~mm}$, desarmador plano), y un gancho para suspender el cadáver.

La prótesis fue implantada en un cadáver donado por del Departamento de Anatomía de la Universidad de Guadalajara. Le fueron removidas dos vértebras: L1 y L2; y fueron reemplazadas con el implante. La columna se expuso mediante un abordaje anterolateral izquierdo y retroperitoneal. Las vértebras fueron identificadas con radiografía. Los discos entre T12-L1 y L2-L3 fueron escindidos. Después, las vértebras L1-L2 fueron removidas con osteotomos y gubias. Una vez hecha la corpectomía, la prótesis fue colocada en su sitio y luego fue expandida rotando el componente $\mathrm{C}$ con una varilla usada como palanca, hasta ejercer compresión en las placas terminales de T12 y L3. Una vez hecho esto, la prótesis fue fijada con dos tornillos esponjosa 6.5 mm rosca corrida a T12 y L3. Luego, el cadáver fue levantado mediante un gancho anclado directamente de la prótesis, hasta quedar completamente suspendido. Mientras el cadáver estaba colgado se le tomaron radiografías en proyección AP del sitio de la corpectomía (Figura 5). Posteriormente el cadáver fue descolgado y luego sometido a flexión, rotación y extensión por seis estudiantes de medicina mientras al mismo tiempo se observaba el comportamiento in situ del implante.

\section{Técnica quirúrgica para prueba en paciente}

En todos los casos los instrumentos usados para la corpectomía y la colocación de la prótesis incluyó un set de osteotomos, cucharillas, gubias, desarmador hexagonal 


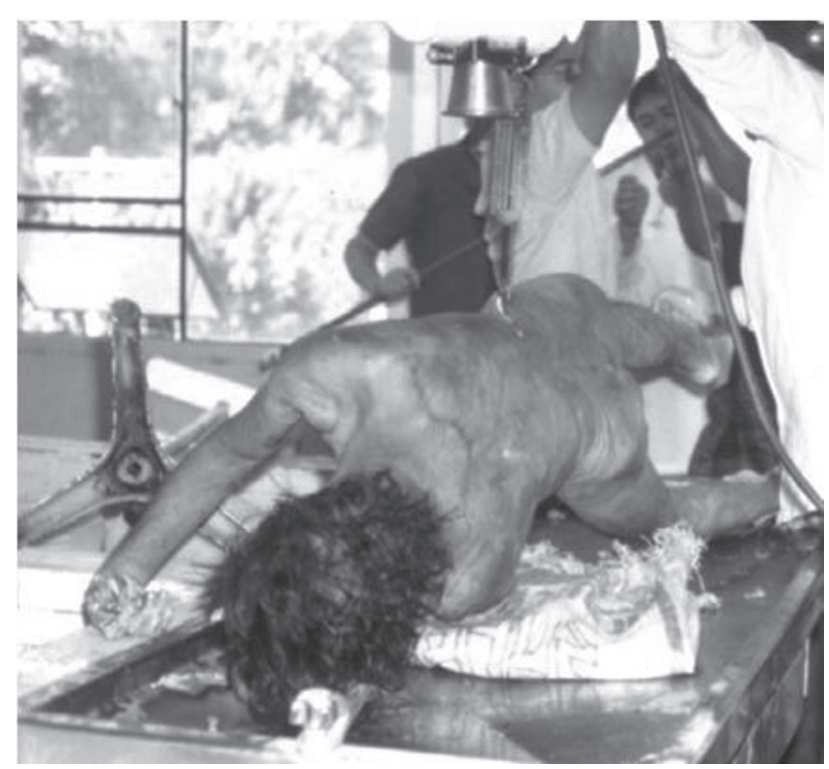

Figura 5

Prueba de fijación en cadáver donde se muestra éste suspendido directamente anclado de la prótesis (A).

Radiografía de cadáver con la prótesis colocada (B).

$4.5 \mathrm{~mm}$, punzón, martillo, impactador de injerto, base para prótesis y un embudo para injerto óseo; los tres últimos diseñados por el primer autor de este artigo.

Se obtuvo consentimiento informado por parte del paciente, una mujer de 50 años de edad que dos años previos a su ingreso le fue removido un cáncer de tiroides. Además, el Comité de Ética del hospital aprobó el procedimiento quirúrgico y la realización del ensayo en pacientes, una vez que se presentó la información sobre las pruebas pre-clínicas.

Previo a la intubación para anestesia general, todas las vías venosas y cateterizaciones necesarias fueron puestas, incluyendo sonda urinaria tipo Foley y un catéter epidural para control del dolor postoperatorio. La paciente fue colocada en decúbito lateral. Una mesa quirúrgica radiolúcida fue utilizada y la patología dictó el sitio del abordaje (anterolateral izquierdo, transpleural y retroperitoneal). La paciente fue asegurada en la mesa de operaciones usando tela adhesiva sobre sus hombros y el trocánter mayor. Todos los puntos de presión fueron almohadillados en forma adecuada. El brazo fue acomodado sobre una mesa adecuadamente. Una vez expuesta la columna, el nivel lesionado fue identificado con fluoroscopia (Figura 6). Después de esto, los vasos segmentarios de las vértebras infra y suprayacente al nivel afectado fueron identificados, ligados y seccionados, luego, con disección subperióstica los cuerpos vertebrales fueron expuestos para colocar dos separadores de Bennett entre los cuerpos vertebrales y los grandes vasos.

Posteriormente, usando un bisturí y con dirección de anterior a posterior, los discos supra e infrayacente a la vértebra lesionada fueron seccionados, después, con gubia y pinza de disco fueron removidos en su totalidad. Enseguida

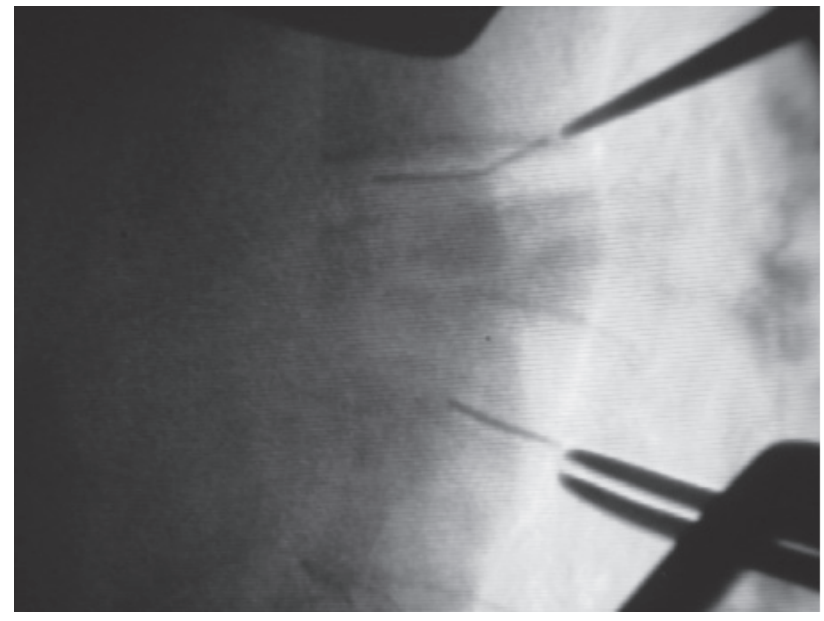

Figura 6

Identificación de vértebra patológica mediante fluoroscopia.

el pedículo de la vértebra tumoral fue identificado y cortado con osteotomo. La vértebra tumoral fue removida con un osteotomo, gubias o cucharillas. Finalmente, una cucharilla curva fue usada para remover los fragmentos que comprimen la médula espinal o los componentes neurológicos de la cauda equina. Una vez hecha la corpectomía, la prótesis vertebral fue puesta en su base para ser rellenada con el injerto óseo proveniente de la costilla removida del paciente, el cual fue compactado (Figura 7).

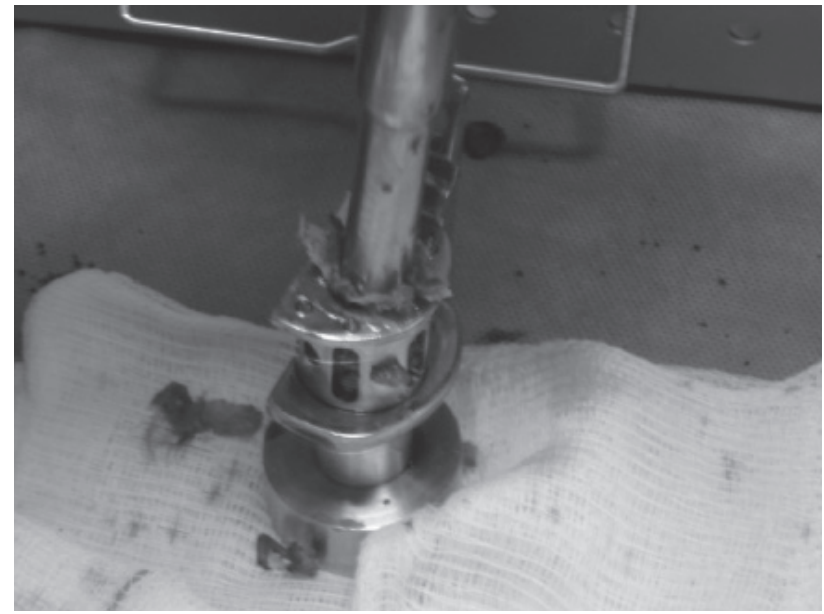

Figura 7

Prótesis vertebral sentada en su base para ser rellenada con injerto óseo, el cual es compactado.

Luego con un reductor de Lowman adaptado, la prótesis fue sujetada para su colocación en el sitio de la corpectomía, entonces el paciente fue hiperextendido y la prótesis colocada. Enseguida se verificó la orientación de la prótesis mediante fluoroscopia (Figura 8). Para expandir la prótesis se rotó el cilindro central (componente $\mathrm{C}$ ) con la palanca de ajuste; así la prótesis corrige la deformidad y da tensión a los tejidos blandos. Una vez expandida, con fluoroscopio se verificó su orientación de nuevo. Posteriormente usando un punzón se hicieron orificios para la fijación con 


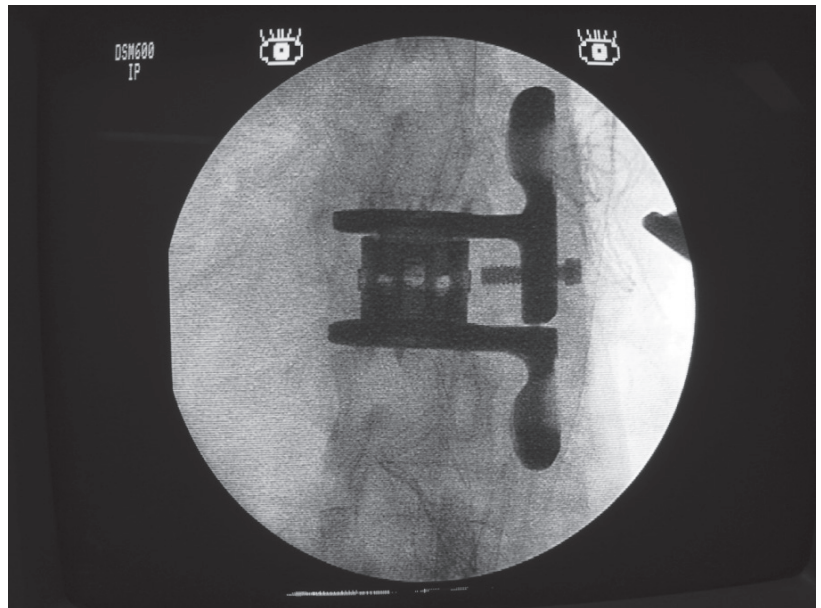

Figura 8

Vista fluoroscópica de la prótesis colocada en buena posición.

tornillos esponjosa de diámetro $6.5 \mathrm{~mm}$ rosca corrida; dos en el segmento proximal y dos en el caudal. Finalmente, la herida quirúrgica fue cerrada por planos.

\section{RESULTADOS}

\section{Prueba en cadáver}

Las radiografías que le fueron tomadas al cadáver no mostraron pérdida de la fijación, a pesar de que el dinamómetro mostró que el cadáver pesaba $60 \mathrm{~kg}$. Esta maniobra de levantar el cadáver generaba momentos de flexión en sentido lateral con brazos de palanca de $80 \mathrm{~cm}$ de largo; por lo cual se calcula que la prótesis fue demandada en su punto más vulnerable con una fuerza aproximadamente de 588 N. En relación a la segunda prueba, la estabilidad se conservó y no se visualizó movimiento del implante. No se pudo calcular la fuerza empleada en estas maniobras.

Basados en este experimento, establecimos la hipótesis de que la función dual de jaula y placa integrada en la prótesis de cuerpo vertebral diseñada ad hoc establece una ventaja mecánica, comparada con la función conjunta de la jaula y de la placa puestas en un segmento pero separadas para la estabilización de la columna y corrección de la deformidad, ya que el diseño de la prótesis permite aplicar fuerza axial y fijación lateral al mismo tiempo, a través de un solo implante. Satisfechos con la fijación y la estabilización lograda en el cadáver, decidimos implantarla al primer paciente de nuestro hospital que tuviera un tumor primario o metastásico en la columna, con dolor intratable, inestabilidad, déficit neurológico y que no respondiera a tratamientos no quirúrgicos

\section{Prueba en paciente}

A una mujer de 50 años de edad que dos años previos a su ingreso le fue removido un cáncer de tiroides, le fue colocada el implante JR. A su ingreso a nuestra institución presentaba dolor axial y radicular asociado a paresia en extremidad inferior izquierda. Los estudios de neuroimagen mostraron una metástasis de L3 (Figura 9). La paciente fue sometida a corpectomía y reemplazo con la prótesis. La cirugía transcurrió sin incidentes o accidentes. El dolor cedió de forma casi inmediata en el postoperatorio. La paciente sobrevivió por siete años haciendo su vida normal, no observándose fallas del implante, hasta que presentó nuevamente dolor lumbar y debilidad de las extremidades inferiores. Se obtuvieron nuevos estudios de neuroimagen y se evidenció recidiva local con compresión severa de cauda equina por la parte posterior, por lo que fue sometida a descompresión posterior e instrumentación con tornillos transpediculares. Al finalizar la cirugía la paciente sufrió paro cardiorrespiratorio y falleció.

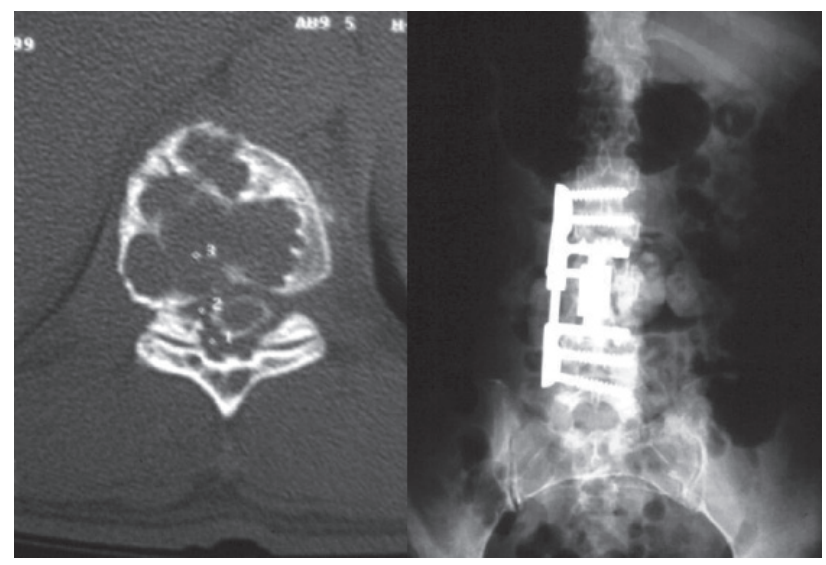

Figura 9

Mujer de 50 años con cáncer metastásico de tiroides en $L 3$, con dolor axial y radicular asociado a paresia, con reemplazo de cuerpo vertebral con el implante JR. Tomografía axial computada de columna lumbar que muestra lesiones líticas y compresión de cauda equina (izquierdo). Radiografía AP de columna lumbar que muestra la prótesis colocada en el sitio de la corpectomía (derecho).

\section{DISCUSIÓN}

La literatura reporta pocas complicaciones relacionadas con los implantes de los sistemas actuales para instrumentación anterior, sin embargo en este grupo se incluyen fracturas de tornillos, pernos y pérdida de la reducción con cifosis progresiva. Kaneda et al. ${ }^{4}$ han reportado que en instrumentaciones anteriores, las complicaciones más frecuentes fueron pseudoartrosis, falla del implante, lesión a grandes vasos, infección y lesión de raíces nerviosas. Kaneda et al. ${ }^{4}$ no observaron en su serie casos de muerte como complicación de la instrumentación anterior.

El concepto de estabilidad de la columna presenta en el contexto de la clínica diferentes connotaciones para los autores. Holdsworth ${ }^{5}$ dividió la columna en anterior y posterior. La columna anterior es compuesta por los discos intervertebrales y el cuerpo vertebral más el ligamento longitudinal anterior, mientras que la columna posterior incluye todas las estructuras posteriores al ligamento longitudinal posterior. De acuerdo con este autor, la estabilidad radica en la integridad del complejo osteoligamentario posterior. Mientras que en el concepto de Denis ${ }^{6}$, la columna anterior de Holdsworth fue dividida en dos, anterior y media. 
Denis ${ }^{6}$ sugirió que la estabilidad radica en la integridad de dos columnas, de acuerdo a este modelo, la destrucción de dos o más es igual a inestabilidad. Kostuik ${ }^{7}$ basó su modelo de estabilidad en el concepto de las tres columnas de Denis, dividiendo la columna en dos mitades: izquierda y derecha. De acuerdo con este modelo, si una o dos de las columnas fueron destruidas, la columna sería estable, pero si son $\geq 3$ sería inestable. Además, de acuerdo con White y Panjabi ${ }^{8}$, la estabilidad clínica se define como la habilidad de la columna para limitar los desplazamientos bajo cargas fisiológicas, de tal manera que no causa daño ni irrita la médula espinal o las raíces nerviosas y, además, evita la incapacidad deformante o el dolor debido a los cambios estructurales.

La laminectomía previamente invocada para descomprimir los elementos neurológicos solamente sirve para desestabilizar aun más la columna, la cual puede llevar a empeorar el déficit neurológico e incrementar más la deformidad. Por otro lado, la cirugía anterior facilita una descompresión directa ya que permite un abordaje al sitio de la compresión y facilita un acceso para remover la fuente de la compresión de los elementos neurológicos. Además, a través de un dispositivo mecánico colocado en el sitio de la corpectomía, esta permite aplicar fuerza axial con ventaja mecánica para corregir la deformidad y mantener la estabilidad ${ }^{9}$. La laminectomía por sí sola, la cual fue el tratamiento de elección hace 20 años, no es mejor que la radioterapia en la descompresión de la médula espinal por tumores ${ }^{10}$. Hall y MacKay ${ }^{11}$ reportaron que la laminectomía causaba mejora en la neurología en el 9\% de los pacientes. Otros han reportado mejoramiento con laminectomía seguido de radioterapia en el 44\% ${ }^{12}$, todavía, se observa mejora neurológica en el $45 \%$ de los pacientes tratados sólo con radioterapia ${ }^{13,14}$.

Helweg-Larson ${ }^{15}$ concluyó que en casos de compresión medular por metástasis, el pronóstico para la deambulación está en relación a la identificación de metástasis antes de que la paresia aparezca. Otros han reportado datos que soportan la cirugía anterior en lesiones anteriores de la columna ${ }^{16,17}$. Müller inicialmente desarrolló la cirugía anterior de la región toracolumbar en la primera década del siglo 20. Carpenter en 1932 describió por primera vez la fusión intercorporea. Después Ito et al. describieron la técnica de la descompresión anterior. Lane y Moore ${ }^{18}$ reportaron su técnica de fusión anterior en la enfermedad degenerativa de disco. Posteriormente, Hodgson y Stock ${ }^{19}$ así como Harmon ${ }^{20}$ describieron la técnica anterior para el manejo de la tuberculosis vertebral. Humphries et al. fueron los primeros en desarrollar las técnicas de estabilización anterior de la cirugía lumbar en $1961^{21}$.

Desde entonces muchos implantes se han desarrollado, algunos todavía están en uso y otros se han descontinuado, como los fijadores de Dunn ${ }^{22}$. En la década de 1960, los abordajes anteriores de la columna torácica y lumbar fueron desarrollados y posteriormente le siguieron las descompresiones anteriores, las fusiones e las instrumentaciones ${ }^{21}$. Muchos cirujanos de escoliosis mostraron que la colocación de implantes para fijaciones anteriores podría ser útil no solamente para aumentar la estabilidad o fusión ósea, sino también para facilitar fuerza para la manipulación, reducción y corrección de la deformidad. Dwyer inicialmente utilizó su cable para fijación, pero después reportó fallas ${ }^{21}$. Zielke subsecuentemente substituyó el cable por una varilla roscada, la cual encontró que era una estructura más estable ${ }^{21}$.

En las décadas de 1970 y 1980, el implante de Harrington-Kostuik apareció para casos de deformidades no escolióticas ${ }^{7}$. Estos implantes fueron puestos anterolateralmente para evitar complicaciones con las estructuras vasculares. Al mismo tiempo Dunn ${ }^{22}$ desarrolló un implante para mejorar la estabilidad de la columna, ya que los anteriores dispositivos se consideraban estructuralmente débiles. Más recientemente el dispositivo de Kaneda fue desarrollado ${ }^{4,21}$. Así como el implante de Harrington-Koustik, el de Kaneda permite la distracción anterior y provee de una fijación estable, lo cual lleva a una integración del injerto óseo. Para ser más estable en rotación, al implante de Kaneda le fueron unidas dos barras accesorias.

Otros implantes anterolaterales incluyen los sistemas de placas tales como la de Armstrong y la Syracuse o Yuan I-beam plate ${ }^{21}$. Danek desarrolló la placa anterior $Z$ plate, la cual tiene capacidad bloqueante ${ }^{21}$. Muchos implantes posteriores habían sido usados en la parte anterior de la columna como el CD system y el TSRH/Danek y el SVN (Sebastian-Abhad system). Todos estos implantes (varillas y placas) tienen el potencial de fallar como resultado de la reabsorción ósea, lo cual lleva al movimiento del implante y su falla ${ }^{5}$. El implante de Pinto fue uno de los distractores tempranos usados para corregir las deformidades cifóticas $^{8}$. De este concepto, Rezaian ${ }^{8}$ desarrolló un implante que funcionaba como un gato hidráulico para distraer entre dos cuerpos vertebrales. Sin embargo, el implante de Rezaian era menos estable en rotación que las placas. Por otro lado, jaulas cilíndricas de titanio se desarrollaron para ser colocadas entre cuerpos vertebrales y ser rellenadas con injerto óseo ${ }^{7,21}$. Estos sistemas actuaban como espaciadores estructurales, mientras que el injerto óseo que estaba dentro de ellas se incorporaba. Empero, estos implantes son inestables en rotación y requieren de una fijación adicional (placa lateral para aumentar su estabilidad) ${ }^{7}$.

En menos palabras, cuando las instrumentaciones anteriores se desarrollaron para fracturas o tumores había dos implantes básicos: placas y varillas. Aunque estos sistemas en pruebas biomecánicas resistían bien al momento de flexión, eran débiles en rotación, por eso, para contrarrestar esta deficiencia, uniones cruzadas a las barras fueron usadas para lograr el efecto biomecánico de una placa ${ }^{23}$. Además, las fijaciones anteriores que dependen de tornillos a través de los cuerpos vertebrales están sujetas a una incidencia mayor de fractura, no solo por su forma de fijación a la vértebra en ángulo recto, sino como consecuencia 
de la reabsorción del injerto óseo, lo cual resulta en falla del implante por fatiga ${ }^{23}$. Otra vez, para contrarrestar esta desventaja, implantes intercorpóreos (jaulas) fueron diseñados para la reconstrucción, sin embargo estos implantes son inestables en rotación ${ }^{21}$. Una placa estabiliza en rotación pero es insuficiente en flexión y una jaula estabiliza en flexión, pero es insuficiente en rotación. Entonces, para estabilizar la columna en todos los planos, una placa necesita una jaula o viceversa.

El prototipo diseñado de la prótesis de cuerpo vertebral JR no permitía aplicar fuerza axial para corregir deformidad en cifosis o mucho menos para ser usado en casos donde fuera necesario reemplazar dos o tres segmentos. El segundo prototipo se diseñó modular y expandible. Este segundo dispositivo inicialmente funcionaba como placa lateral, pero no funcionaba adecuadamente como jaula cilíndrica. Para hacer la función de jaula expandible, se hizo un orificio central a los tres componentes y así nació el tercer dispositivo con función de jaula y placa lateral integrada en un solo implante. La actual prótesis JR de cuerpo vertebral por su diseño modular y expandible, su modo de fijación, y su localización anterior y lateral (compatible con los grandes vasos) permite ejercer fuerza axial en distracción para corregir cifosis, con ventaja mecánica y fijación lateral a través de la placa. Por otro lado, los elementos horizontales de ambos componentes de la prótesis presentan aproximadamente el mismo radio de las placas terminales de los cuerpos vertebrales, lo cual recrea el momento inercial aumentando de esta forma no sólo la resistencia a la inclinación, sino también la duración del implante. Además, por su apoyo periférico y orificio central a la vez que impide su hundimiento, facilita el contacto biológico mediante el rellenado de la prótesis con injerto óseo. Por otro lado, debido a que los tornillos para la fijación lateral no soportan carga axial en compresión, no están sujetos a fatiga mecánica, por lo cual su riesgo de fractura por estrés es casi nulo. Por todo lo anterior, nuestro implante tiene ventajas sobre las jaulas, las placas o las barras, ya que en estos sistemas no pueden estabilizar la columna por sí solos. Se necesitan usualmente al menos dos dispositivos para lograr la estabilización mecánica.

Aunque en principio la prótesis JR fue diseñada para tumores de cuerpo vertebral, actualmente también la usamos en fracturas toracolumbares por explosión, con o sin daño neurológico, además en cifosis ya sea postraumática o tuberculosa.

\section{CONCLUSIÓN}

La prótesis vertebral JR es una opción viable para estabilizar la columna después de corpectomía en pacientes con déficit neurológico, dolor intratable e inestabilidad causada por una lesión primaria o metastásica. Las evidentes funciones de jaula y placa constituyen una ventaja mecánica para estabilizar la columna después de corpectomía. Nuevos estudios son necesarios para ampliar la experiencia y corroborar nuestras conclusiones.

\section{REFERENCIAS}

1. Harrington PR. The history and development of Harrington instrumentation. Clin Orthop Relat Res. 1988;227:3-5.

2. Luque ER. The anatomic basis and development of segmental spinal instrumentation. Spine. 1982;7(3):256-9.

3. Lucas GL, Cooke FW, Friis EA. A primer of biomechanics. New York: Springer; 1998. p. 59.

4. Kaneda K, Taneichi H, Abumi K, Hashimoto T, Satoh S, Fujiya $\mathrm{M}$. Anterior decompression and stabilization with the Kaneda device for thoracolumbar burst fractures associated with neurological deficits. J Bone Joint Surg Am. 1997;79(1):69-83.

5. Holdsworth F. Fractures, dislocations, and fracture-dislocations of the spine. J Bone Joint Surg Am. 1970;52(8):1534-51.

6. Denis F. The three column spine and its significance in the classification of acute thoracolumbar spinal injuries. Spine. 1983;8(8):817-31.
7. Kostuik JP. Anterior fixation for burst fractures of the thoracic and lumbar spine with or without neurological involvement. Spine. 1988;13(3):286-93.

8. White AA III, Panjabi MM. Clinical biomechanics of the spine. 2 nd ed. Philadelphia: Lippincott; c1990.

9. Bell GR. Surgical treatment of spinal tumors. Clin Orthop Relat Res. 1997;(335):54-63. Review.

10.Gilbert HA, Kagan AR, Nussbaun H, Rao AR, Satzman J, Chan P, et al. Evaluation of radiation therapy for bone metastases: pain relief and quality of life. AJR Am J Roentgenol. 1977;129(6):1095-6.

11.Hall AJ, Mackay NN. The results of laminectomy for compression of the cord or cauda equina by extradural malignant tumour. J Bone Joint Surg. 1973;55(3):497-505.
12. Young RF, Post EM, King GA. Treatment of spinal epidural metastases. Randomized prospective comparison of laminectomy and radiotherapy. J Neurosurg. 1980;53(6):741-8.

13.Bach F, Larsen BH, Rohde K, Børgesen SE, Gjerris F, BøgeRasmussen T, et al. Metastatic spinal cord compression. Occurrence, symptoms, clinical presentations and prognosis in 398 patients with spinal cord compression. Acta Neurochir (Wien). 1990;107(1-2):37-43.

14.Gilbert RW, Kim JH, Posner JB. Epidural spinal cord compression from metastatic tumor: diagnosis and treatment. Ann Neurol. 1978;3(1):40-51.

15.Helweg-Larsen S. Clinical outcome in metastatic spinal cord compression. A prospective study of 153 patients. Acta Neurol Scand. 1996;94(4):269-75. 
16. Sundaresan N, Galicich JH, Lane JM, Bains MS, McCormack P. Treatment of neoplastic epidural cord compression by vertebral body resection and stabilization. J Neurosurg. 1985;63(5):676-84.

17.Siegal T, Tiqva P, Siegal T. Vertebral body resection for epidural compression by malignant tumors. Results of forty-seven consecutive operative procedures. J Bone Joint Surg Am. 1985;67(3):375-82.

18.Lane JD Jr, Moore ES Jr. Transperitoneal approach to the intervertebral disc in the lumbar area. Ann Surg. 1948;127(3):537-51.
19.Hodgson AR, Stock FE. Anterior spinal fusion a preliminary communication on the radical treatment of Pott's disease and Pott's paraplegia. Br J Surg. 1956;44(185):266-75.

20.Harmon PH. Anterior extraperitoneal lumbar disc excision and vertebral body fusion. Clin Orthop. 1960;18:169-98.

21.Carl AL, Roger DJ. Advances in spinal instrumentation: a review article. Semin Spine Surg. 1997;9:204-26.

22.Dunn HK. Anterior spine stabilization and decompression for thoracolumbar injuries. Ortop Clin North Am. 1986;17(1):113-9.
23.Metastatic tumors of the spine: diagnosis and treatment. J Am Acad Orthop Surg. 1993;1(2):76-86.

\section{Correspondencia}

Juan José Ramírez Jiménez

Servicio de Ortopedia de Hospital Civil de Guadalajara "Fray Antonio Alcalde". Hospital 278, Col. El Retiro.

C.P.: 44280 - Guadalajara, Jalisco, México.

Tel: +52 (33) 3613-3951

Fax: +52 (33) 3613-3951

E-mail: raij0709@hotmail.com 